\title{
Occurrence of myelodysplastic syndrome during paclitaxel- and carboplatin-based chemotherapy for recurrent ovarian cancer: Case report ${ }^{*}$
}

\author{
Hiroshi Takagi ${ }^{1}$, Satoshi Ichigo ${ }^{1}$, Kazutoshi Matsunami ${ }^{1}$, Haruna Takagi ${ }^{1}$, Tsuneko Ikeda ${ }^{2}$, \\ Takayuki Murase $^{2}$, Atsushi Imai ${ }^{1 \#}$ \\ ${ }^{1}$ Department of Obstetrics and Gynecology, Matsunami General Hospital, Gifu, Japan \\ ${ }^{2}$ Department of Pathology, Matsunami General Hospital, Kasamatsu, Gifu, Japan \\ Email: "
}

Received 1 February 2013; revised 2 March 2013; accepted 10 March 2013

\begin{abstract}
Platinum and taxane have significant activity as key drugs in current treatment of advanced ovarian cancer. While myelodysplastic syndrome (MDS) and acute leukemia are well-known secondary disease after administration of chemotherapy, particularly with alkylating agents, they have only rarely been reported in the context of ovarian cancer treatment. In this case report a 59-year-old Japanese developed a MSD during ongoing second induction chemotherapy with carboplatin and paclitaxel for recurrent ovarian cancer. Her second induction began 9 months after completion of her first course of chemotherapy. Cytogenetic analyses showed typical chromosomal aberration. Although the finding of pancytopenia is also seen during chemotherapy without MSD/acute leukemia, bone marrow aspiration and biopsy should be considered to confirm the clinical suspicion of the lifethreatening disease when encountered persistent pancytopenia following carboplatin- and paclitaxel-based chemotherapy in an ovarian cancer patient.
\end{abstract}

Keywords: Chemotherapy-Related Myelodysplastic Syndrome; Ovarian Cancer; Platinum; Taxane

\section{INTRODUCTION}

Cytotoxic chemotherapeutic agents shows a marked activity in the treatment of a variety of types of cancers including endometrial and ovarian cancers and improve the overall survival rate of the patients [1,2]. Myelodysplastic syndrome (MDS) is a clonal hematopoietic disorder that may be caused by cytotoxic chemotherapy with alkylating agents and topoisomerase inhibitors and/ or radiation therapy [3-5]. A extensive review of reported

\footnotetext{
"Conflict of interest: the authors have no conflict of interest to disclose. "Corresponding author.
}

therapy-related MDS rates concluded that up to $10 \%$ of patients treated with conventional dose alkylator-based regimens or high dose therapy develop MDS within a decade of primary therapy [6]. Comparable changes in the course of treatment with taxane and platinum for ovarian cancer have only rarely reported $[7,8]$ and in these cases an interval of over 20 months has been observed between treatment and onset of the secondary disease. We report an interesting case of treatment-related MSD in a patient with ovarian cancer who received carboplatin and paclitaxel for recurrent ovarian cancer.

\section{CASE REPORT}

A 59-year-old woman diagnosed with ovarian cancer in February 2011 was treated with radical surgery and adjuvant chemotherapy. A total abdominal hysterectomy, bilateral salpingo-oophorectomy, pelvic lymph node dissection and omentectomy were performed. The washing peritoneal cytology was reported positive. A histopathologic diagnosis was serous papillary adenocarcinoma, grade 3 (Figure 1(a)). She was staged as FIGO stage IIIb, and submitted to six courses of systemic chemotherapy (paclitaxel $\left(175 \mathrm{mg} / \mathrm{m}^{2}\right.$ ) and carboplatin (AUC 5).

Nine months after the first adjuvant therapy, the levels of CA19-9 and CA125 gradually became unstable and moderately high and peritoneal dissemination on CT scan was found. The patient was started on additional cycles of systemic paclitaxel- and carboplatin-based chemotherapy. Prior to cycle 5, she was found to be pancytopenic (WBC count of $2740 \mu \mathrm{l}$, RBC $2.65 \times 10^{6} / \mu 1$, platelets $5.8 \times 10^{4} / \mu \mathrm{l}$, hemoglobin $7.9 \mathrm{~g} / \mathrm{dl}, \mathrm{MCV} 92.1 \mathrm{fl}$, $\mathrm{MCH} 29.5 \mathrm{pg}$, MCHC 32.1\%) with the appearance of nucleated red cells, schistocytes, poikilocytosis, and polychromasis on a peripheral blood smear. A bone marrow biopsy in September 2012 showed a hypocellular marrow with relative erythroid hyperplasia, marked megaloblastic and dysplastic changes, a left-shifted myeloid series, consistent with myelodysplasia (Figure 1(b)). 


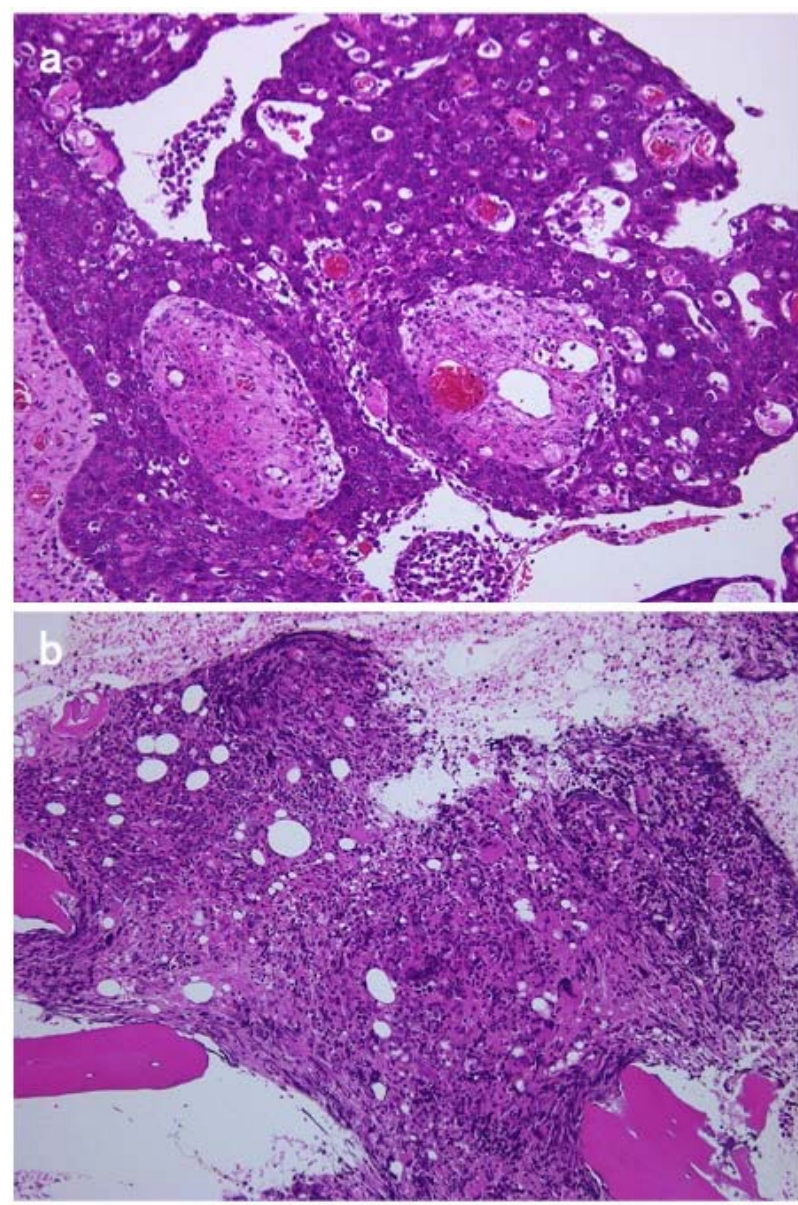

Figure 1. Photomicrographs showing serous papillary adenocarcinoma (a) and myelodysplasia (b) (hematoxylin-eosin, 100×).

Cytogenic studies demonstrated 10 of 17 normal metaphases and 2 abnormal clones $(46, \mathrm{XX}, \operatorname{der}(5) \mathrm{t}(5 ; 15)(\mathrm{q} 13$; q15), $-7,+8,-15$, add(17)(p11.2) in 4 metaphases and 46, idem, $-\mathrm{rl},+\mathrm{r} 2$ in 3 ). These findings were suggestive of chemotherapy-related myelodyspla stic syndrome. Peritonitis carcinomatosa progression led the patient to decide to have no further systemic chemotherapy and operation.

\section{DISCUSSION}

We report the occurrence of MDS in a patient with recurrent ovarian cancer during second induction chemotherapy with carboplatin and paclitaxel. The time course of agents is depicted in Figure 2. We believe that carboplatin and/or paclitaxel contributed likely to the accumulation of alkylator-related DNA damage, as the unusually severe myelosuppression during the additional chemotherapy may have reflected a predisposition to the myelotoxic effects of chemotherapy. Although the potential contribution of these two to the induction of MDS is controversial, only few cases of the secondary diseases, particularly MDS and acute myeloblastic leukemia fol-

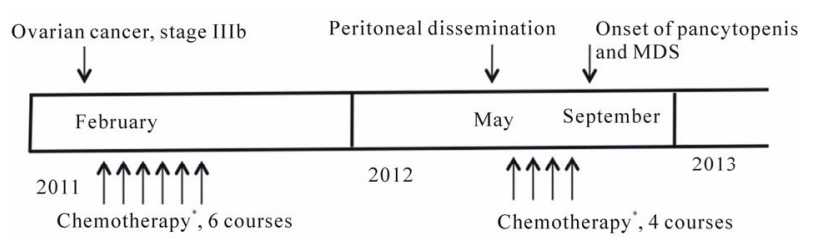

Figure 2. Time line of events from initial diagnosis of ovarian cancer to the onset of myelodisplastic syndrome. ${ }^{*}$ Carboplatinand paclitaxel-based chemotherapy.

lowing ovarian cancer therapy are reported in the literature and in those cases only carboplatin and paclitaxel were used as primary chemotherapy $[7,8]$. The individual contribution of each treatment modality remains undetermined in this patient, although the exposure to carboplatin and/or paclitaxel may have played a role in the development of therapy-related MDS.

Therapy-related MDS/AML has been described following cytotoxic chemotherapy of malignant tumors with classic alkylating agents, including nitrosoureas or procarbazin, radiation therapy alone, or combined chemotherapy and radiotherapy [4,9-11]. The risk rises with increasing age, and there no known factors other than age and duration of therapy to predict which patients might be at higher risk of the secondary diseases. In a review including 28 cases reports of the secondary diseases, the median latency between the start of therapy and the diagnosis of therapy-related MDS/AML was 2.5 years (range, 8 months to 4.5 years) [9]. Our patient with recurrent ovarian cancer developed MDS during additional chemotherapy. However, this patient had previously received six courses of same agents. As Noronha et $a l$. [10] described previously, the clinical feature of therapy-related MDS are result of bone marrow failure. Symptomatic anemia is the most common presentation, but easy bruising and repeated infections may also be prominent. The complete blood count revealed persistent or worsening pancytopenia. These findings are also seen during chemotherapy without MSD/acute leukemia. Bone marrow aspiration and biopsy are reformed to confirm the clinical suspicion of the secondary disease.

The development of MDS is related to the specific DNA-damaging agents, dose, therapy duration, and patient age. The cause of the secondary disease related to carboplatin and paclitaxel remains to be determined. It is unlikely that this is a direct effect of carboplatin and paclitaxel as the phenomenon have not been reported in other diseases treated with these two agents. Taxanes inhibit the disassembly of microtubles which are involved in mitosis and axoplasmic transport [1]. The platinum complexes react in vivo, binding to and causing crosslinking of DNA, which ultimately triggers apoptosis (programmed cell death). Thus these two are unlikely incorporated into DNA to interfere with DNA repair mechanisms. The carboplatin and/or paclitaxel-induced 
event may represent evidence of genomic damage to the bone marrow that may be disposed to multiple genetic changes.

\section{REFERENCES}

[1] Guastalla, J. and Diéras, V. (2003) The taxanes: Toxicity and quality of life considerations in advanced ovarian cancer. British Journal of Cancer, 89, S16-S22. doi:10.1038/sj.bjc.6601496

[2] Hagiwara, H. and Sunada, Y. (2004) Mechanism of taxane neurotoxicity. Breast Cancer, 11, 82-85. doi:10.1007/BF02968008

[3] Smith, S., Le Beau, M., Huo, D., Karrison, T., Sobecks, R., Anastasi, J., Vardiman, J., Rowley, J. and Larson, R. (2003) Clinical-cytogenetic associations in 306 patients with therapy-related myelodysplasia and myeloid leukemia: The University of Chicago series. Blood, 102, 43-52. doi:10.1182/blood-2002-11-3343

[4] Tam, C., Seymour, J., Prince, H., Kenealy, M., Wolf, M., Januszewicz, E. and Westerman, D. (2006) Treatmentrelated myelodysplasia following fludarabine combination chemotherapy. Haematologica, 91, 1546-1450.

[5] Whang-Peng, J., Young, R., Lee, E., Longo, D., Schechter, G. and De Vita, V.J. (1988) Cytogenetic studies in patients with secondary leukemia/dysmyelopoietic syndrome after different treatment modalities. Blood, 71, 403-414.

[6] Armitage, J., Carbone, P., Connors, J., Levine, A., Bennett, J. and Kroll, S. (2003) Treatment-related myelodysplasia and acute leukemia in non-Hodgkin's lymphoma patients. Journal of Clinical Oncology, 21, 897-906. doi:10.1200/JCO.2003.07.113

[7] Abe, A., Ikawa, H., Muguruma, H. and Maeda, Y. (2008) Secondary myelodysplasia and leukemia following carboplatin and paclitaxel-containing chemotherapy for ovarian cancer. (Article in Japanese), Gan To Kagaku Ryoho, 35, 1795-1798.

[8] Yeasmin, S., Nakayama, K., Ishibashi, M., Oride, A., Katagiri, A., Purwana, I., Iida, K., Nakayama, N., Ishikura, H. and Miyazaki, K. (2008) Therapy-related myelodysplasia and acute myeloid leukemia following paclitaxeland carboplatin-based chemotherapy in an ovarian cancer patient: A case report and literature review. International Journal of Gynecologic Cancer, 18, 1371-1376. doi:10.1111/j.1525-1438.2007.01185.x

[9] Perry, J., Brown, M. and Gockerman, J. (1998) Acute leukemia following treatment of malignant glioma. Journal of Neurooncology, 40, 39-46. doi:10.1023/A:1006175831785

[10] Noronha, V., Berliner, N., Ballen, K., Lacy, J., Kracher, J., Baehring, J. and Henson, J. (2006) Treatment-related myelodysplasia/AML in a patient with a history of breast cancer and an oligodendroglioma treated with temozolomide: Case study and review of the literature. Neurological Oncology, 8, 280-283. doi:10.1215/15228517-2006-003

[11] Jäger, G., Höfler, G., Linkesch, W. and Neumeister, P. (2004) Occurrence of a myelodysplastic syndrome (MDS) during first-line 2-chloro-deoxyadenosine (2-CDA) treatment of a low-grade gastrointestinal MALT lymphoma. Case report and review of the literature. Haematologica, 89, ECR1-3. 